Pacific Journal of Mathematics

SHEAR DISTALITY AND EQUICONTINUITY

Fe RIGGi aNd Neson Groh Marki 


\title{
SHEAR DISTALITY AND EQUICONTINUITY
}

\author{
Dennis F. De Riggi and Nelson G. Markley
}

Let $X$ be a compact Hausdorff space, let $\boldsymbol{R}^{p}$ be $p$ dimensional Euclidean space, and let $\left(X, R^{p}\right)$ be a minimal transformation group. It may happen that $\overline{x H}$ will always contain flow lines in at least one direction in $R^{p}$ for any discretr syndetic subgroup $H$ no matter how sparce. We interpret this phenomena as some intrinsic shearing motion in the minimal transformation group. This is quantified in Section 1 and it turns out that equicontinuous minimal sets have as little shear as possible. Since distality is also a rigidity condition, it is natural to investigate the shear of a distal minimal set. We show by example in Section 2 that distal minimal sets can contain more shear than equicontinuous ones.

In Section 3 we show how the topology of $\overline{x H}$ is locally determined by local sections and subspaces of $R^{p}$. Using this result we prove in Section 4 that a distal minimal action of $R^{n-1}$ with trivial isotropy on a compact $n$-dimensional manifold is equicontinuous.

This paper contains portions of the first author's dissertation [1] and generalizations of some results in an unpublished preprint [6] by the second author.

1. Shear. Let $X$ be a compact Hausdorff space and let $\left(X, \boldsymbol{R}^{p}\right)$ be a minimal transformation group. Set $I_{x}=\left\{v \in \boldsymbol{R}^{P}: x v=x\right\}$ and note that it is a closed subgroup of $\boldsymbol{R}^{p}$ which is independent of $x$ because of the minimality. This group will be denoted by $I$ or $I\left(X, \boldsymbol{R}^{p}\right)$ and we will say that $\left(X, \boldsymbol{R}^{p}\right)$ has trivial isotropy when $I=\{0\}$. We will frequently need to assume that $\left(X, \boldsymbol{R}^{p}\right)$ is locally free; that is, given $x \in X$ there exists a neighborhood $W$ of 0 in $\boldsymbol{R}^{p}$ such that $x v \neq x$ for all $v$ in $W$. Clearly $\left(X, \boldsymbol{R}^{p}\right)$ is locally free if and only if $I$ is discrete.

Let $H$ be a closed syndetic (co-compact) subgroup of $\boldsymbol{R}^{p}$. It is well known that $(X, H)$ is pointwise almost periodic and $H_{x}=\{v: x v \in \overline{x H}\}$ is also a closed syndetic subgroup of $\boldsymbol{R}^{p}$ such that $\overline{x H}_{x}=\overline{x H}$. [4, Theorem 4.04, Lemma 2.09 and Lemma 2.10.] Again by minimality $H_{x}$ is independent of $x$. We will say that $H$ is self-enveloping when $x v \in \overline{x H}$ implies $v \in H$ or $H_{x}=H$ for all $x$. When $\overline{x H}=X$ for every closed syndetic subgroup of $\boldsymbol{R}^{p},\left(X, \boldsymbol{R}^{p}\right)$ is said to be totally minimal. Let $\mathscr{S}=\mathscr{S}\left(X, R^{p}\right)$ denote the collection of closed syndetic subgroups of $R^{p}$ which are self-enveloping for $\left(X, \boldsymbol{R}^{p}\right)$. It is obvious that $\left(X, \boldsymbol{R}^{p}\right)$ is totally minimal if and only if $\mathscr{S}=\left\{\boldsymbol{R}^{p}\right\}$. 
If $H$ is a closed subgroup of $\boldsymbol{R}^{p}$, then $H=V \oplus D$ where $V$ is a subspace and $D$ is a discrete subgroup. By the dimension of $H$ we shall always mean the dimension of $V$. We now define the shear of $\left(X, \boldsymbol{R}^{p}\right)$ as follows:

$$
s=s\left(X, \boldsymbol{R}^{p}\right)=\min \left\{\operatorname{dim} H: H \in \mathscr{S}\left(X, R^{p}\right)\right\}
$$

Note that $s\left(X, \boldsymbol{R}^{p}\right)=p$ is equivalent to $\left(X, \boldsymbol{R}^{p}\right)$ is totally minimal. Consequently $s=s\left(X, \boldsymbol{R}^{p}\right)$ is a measure of how near $\left(X, \boldsymbol{R}^{p}\right)$ is to being totally minimal. We will be primarily interested in the antithesis of total minimality, $s\left(X, \boldsymbol{R}^{p}\right)=0$. Since $I$ is contained in $H$ for every $H \in \mathscr{S}, s$ can not be zero when the action is not locally free; that is, $\operatorname{dim} I \leqq s$.

Proposition 1.1. Let $\varphi:\left(X, \boldsymbol{R}^{p}\right) \rightarrow\left(Y, \boldsymbol{R}^{p}\right)$ be a homomorphism. Then $s\left(X, \boldsymbol{R}^{p}\right) \leqq s\left(Y, \boldsymbol{R}^{p}\right)$.

Proof. If suffices to show that $\mathscr{S}\left(Y, \boldsymbol{R}^{p}\right) \subset \mathscr{S}\left(X, \boldsymbol{R}^{p}\right)$. Let $H \in$ $\mathscr{S}\left(Y, \boldsymbol{R}^{p}\right)$ and suppose that $x v \in \overline{x H}$ for some $x \in X$. Then $\varphi(x) v \in$ $\varphi(x) H$ implies $v \in H$ and $H \in \mathscr{S}\left(X, \boldsymbol{R}^{p}\right)$.

Proposition 1.2. Let $H$ be a closed syndetic subgroup of $\boldsymbol{R}^{p}$. Then $H \in \mathscr{S}\left(X, \boldsymbol{R}^{p}\right)$ if and only if there exists an equicontinuous factor $\left(Y, \boldsymbol{R}^{p}\right)$ of $\left(X, \boldsymbol{R}^{p}\right)$ such that $I\left(Y, \boldsymbol{R}^{p}\right)=H$.

Proof. If $H \in \mathscr{S}\left(X, \boldsymbol{R}^{p}\right)$, then $\overline{x H}=\overline{y H}$ defines a closed invariant equivalence relation on $X\left[4\right.$, Theorem 2.32]. Let $\left(Y, \boldsymbol{R}^{p}\right)$ be the resulting flow on this quotient space. If $[x]$ denotes the point in $Y$ determined by $x \in X$, then $[x] v=[x]$ iff $\overline{x v H}=\overline{x H}$ iff $x v \in \overline{x H}$ iff $v \in H$. Clearly $\left(Y, \boldsymbol{R}^{p}\right)$ is equicontinuous because $\boldsymbol{R}^{p} / H$ is compact. For the converse note that $I\left(Y, \boldsymbol{R}^{p}\right)=H$ implies $H \in \mathscr{S}\left(Y, \boldsymbol{R}^{p}\right) \subset \mathscr{S}\left(X, \boldsymbol{R}^{p}\right)$.

Proposition 1.3. If $\left(X, \boldsymbol{R}^{p}\right)$ is equicontinuous, then $s\left(X, \boldsymbol{R}^{p}\right)=$ $\operatorname{dim} I\left(X, \boldsymbol{R}^{p}\right)$.

Proof. Without loss of generality we can assume that $\operatorname{dim} I=0, X$ is a topological group, $\theta: \boldsymbol{R}^{p} \rightarrow X$ is a continuous homomorphism, and $x v=x+\theta(v)$. Let $\chi_{1}$ be a character on $X$ such that $\chi_{1}\left(\theta\left(f_{1}\right)\right) \neq 1$ for some $f_{1} \neq 0$ in $\boldsymbol{R}^{p}$. Then exists a linear map $T_{1}: \boldsymbol{R}^{p} \rightarrow \boldsymbol{R}$ such that $\exp \left(2 \pi i T_{1}(v)\right)=\chi_{1}(\theta(v))$. Now we can choose $f_{2} \in \operatorname{ker}\left(T_{1}\right)$ and $\chi_{2}$ such that $\chi_{2}\left(\theta\left(f_{2}\right)\right) \neq 1$ and then $f_{3} \in \operatorname{ker} T_{1} \cap \operatorname{ker} T_{2}$ with $\chi_{3}\left(\theta\left(f_{3}\right)\right) \neq 1$. Let $\psi: X \rightarrow \boldsymbol{K}^{p}$ by $\psi(g)=\left(\chi_{1}(g), \cdots, \chi_{p}(g)\right)$ where $\boldsymbol{K}=\{z \in \boldsymbol{C}:|z|=1\}$ and let $T$ be the lift of $\psi \circ \theta$ to $\boldsymbol{R}^{p}$. Then it follows that the matrix of $T$ with 
respect to $\left\{f_{1}, \cdots, f_{p}\right\}$ and the standard basis is triangular with nonzero diagonal entries. Therefore, $T$ and hence $\psi \circ \theta$ are onto. It is now easy to check that $H=\operatorname{ker}(\psi \circ \theta) \in \mathscr{S}\left(X, \boldsymbol{R}^{p}\right)$ and $\operatorname{dim} H=0$.

Proposition 1.4. The transformation group $\left(X, \boldsymbol{R}^{p}\right)$ is not totally minimal if and only if $\left(X, \boldsymbol{R}^{p}\right)$ has a nontrivial equicontinuous factor.

Proof. Suppose $H \in \mathscr{S}$ such that $H \neq \boldsymbol{R}^{p}$. Then the factor $\left(Y, \boldsymbol{R}^{p}\right)$ such that $I\left(Y, \boldsymbol{R}^{p}\right)=H$ is nontrivial and equicontinuous because the compact group $\boldsymbol{R}^{p} / H$ acts on $Y$ with trivial isotropy. Conversely if $\left(Y, \boldsymbol{R}^{p}\right)$ is a nontrivial equicontinuous factor, then $s\left(X, \boldsymbol{R}^{p}\right) \leqq s\left(Y, \boldsymbol{R}^{p}\right) \leqq$ $p-1$ which implies $\left(X, \boldsymbol{R}^{p}\right)$ is not totally minimal.

Proposition 1.5. Let $\left(E, \boldsymbol{R}^{p}\right)$ be the maximal equicontinuous factor of $\left(X, \boldsymbol{R}^{p}\right)$. Then $s\left(X, \boldsymbol{R}^{p}\right)=s\left(E, \boldsymbol{R}^{p}\right)=\operatorname{dim} I\left(E, \boldsymbol{R}^{p}\right)$.

Proof. Let $H$ be an element of $\mathscr{S}\left(X, \boldsymbol{R}^{p}\right)$ such that $\operatorname{dim} H=$ $s\left(X, \boldsymbol{R}^{p}\right)$ and let $\left(Y, \boldsymbol{R}^{p}\right)$ be a factor of $\left(X, \boldsymbol{R}^{p}\right)$ with $H=I\left(Y, \boldsymbol{R}^{p}\right)$. Since $\left(Y, \boldsymbol{R}^{p}\right)$ is equicontinuous, we have

$$
\operatorname{dim} H=s\left(X, \boldsymbol{R}^{p}\right) \leqq s\left(E, \boldsymbol{R}^{p}\right) \leqq s\left(Y, \boldsymbol{R}^{p}\right)=\operatorname{dim} H .
$$

COROllaRY 1.6. The maximal equicontinuous factor of $\left(X, \boldsymbol{R}^{p}\right)$ is locally free if and only if $s\left(X, \boldsymbol{R}^{p}\right)=0$.

An immediate consequence of this corollary is that $s\left(X, \boldsymbol{R}^{p}\right)=0$ when $\left(X, \boldsymbol{R}^{p}\right)$ is locally free and almost automorphic. However, when you take $\left(X, \boldsymbol{R}^{p}\right)$ to be distal and locally free, things are not so simple.

2. A distal minimal set with nonzero shear. In this section we will construct a distal minimal transformation group $\left(X, \boldsymbol{R}^{3}\right)$ whose maximal equicontinuous factor is the torus $\boldsymbol{K}^{2}$. Then by Proposition $1.5 s\left(X, \boldsymbol{R}^{3}\right)>0$.

Proposition 2.1. Let $\alpha, \beta, \gamma \in K$ such that $\{1, \alpha\}$ and $\{\beta, \gamma\}$ are rationally independent subsets of $\boldsymbol{K}$, and let $S: \boldsymbol{K}^{4} \rightarrow \boldsymbol{K}^{4}$ be given by $S\left(z, w_{1}, w_{2}, w_{3}\right)=\left(\alpha z, z w_{1}, \beta z w_{2}, \gamma z w_{3}\right)$. Then $\left(K^{4}, S\right)$ is minimal and distal.

Proof. Because it is a group extension of $(\boldsymbol{K}, \alpha)$ it is obviously distal. By using a Theorem of Keynes and Newton [5, page 66, Theorem C] to prove minimality, it suffices to show that the only continuous function $\chi: K^{4} \rightarrow \boldsymbol{K}$ of the form

$$
\chi\left(z, w_{1}, w_{2}, w_{3}\right)=w_{1}^{m} w_{2}^{n} w_{3}^{p}
$$


such that $\chi \circ S=\chi$ when $m, n, p \in Z$ is the constant function $\chi \equiv$ 1. This reduces to $z^{m+n+p} \beta^{n} \gamma^{p}=1$ for all $z$ which implies $m+n+p=0$, $n=p=0$, and $m=0$ to complete the proof.

Proposition 2.2. The maximal equicontinuous factor of $\left(\boldsymbol{K}^{4}, S\right)$ is $(\boldsymbol{K}, \alpha)$ under the map $\left(z, w_{1}, w_{2}, w_{3}\right) \rightarrow z$.

Proof. It suffices to show that any pair of points which differ in exactly one of the last three coordinates is in the equicontinuous structure relation. Since the regionally proximal relation coincides with the equicontinuous structure relation because the acting group is abelian [2], this reduces to showing that $(z, w)$ and $\left(z, w^{\prime}\right)$ are regionally proximal in $\boldsymbol{K}^{2}$ under $T(z, w)=(\alpha z, \delta z w)$ where $\delta \in K$. This is a group extension of $(K, \alpha)$ and hence there exists a closed subgroup $G$ of $\boldsymbol{K}$ such that $(z, w)$ and $\left(z, w^{\prime}\right)$ are regionally proximal if and only if $w=w^{\prime} g$ for some $g \in G$. If $G$ is finite, then the maximal equicontinuous factor of $\left(K^{2}, T\right)$ has the form $\left(K^{2}, T^{\prime}\right)$ when $T^{\prime}(z, w)=\left(\alpha z, z^{k} w\right)$ and $k>0$ which is not equicontinuous. Therefore, $G=K$ and the proof is complete.
Define
$\hat{S}: \boldsymbol{K}^{4} \times \boldsymbol{R} \rightarrow \boldsymbol{K}^{4} \times \boldsymbol{R}$
by
$\hat{S}\left(z, w_{1}, w_{2}, w_{3}, x\right)=$ $\left(\alpha z, z w_{1}, \beta z w_{2}, \gamma z w_{3}, x-1\right)$. Let $X$ be the quotient of $\boldsymbol{K}^{4} \times \boldsymbol{R}$ by the cyclic group generated by $\hat{S}$. Then the canonical projection of $\boldsymbol{K}^{4} \times \boldsymbol{R}$ onto $X$ is a regular covering. We define a flow on $\boldsymbol{K}^{4} \times \boldsymbol{R}$ by $\left(\left(z, w_{1}, w_{2}, w_{3}, x\right), t\right) \rightarrow\left(z, w_{1}, w_{2}, w_{3}, x+t\right)$ which induces a flow on $X$ because it commutes with $\hat{S}$. Similarly we define a $\boldsymbol{K}^{3} \times \boldsymbol{R}$ action on $X$ by first defining

$$
\left(\left(z, w_{1}, w_{2}, w_{3}, x\right),\left(\zeta_{1}, \zeta_{2}, \zeta_{3}, t\right)\right) \rightarrow\left(z, w_{1} \zeta_{1}, w_{2} \zeta_{2}, w_{3} \zeta_{3}, x+t\right) .
$$

Finally we define an $\boldsymbol{R}^{3}$ action $X$ by mapping $\boldsymbol{R}^{3} \rightarrow \boldsymbol{K}^{3} \times \boldsymbol{R}$ by $\left(t_{1}, t_{2}, t_{3}\right) \rightarrow\left(\varphi\left(t_{1}, t_{2}\right), t_{3}\right)$ when $\varphi$ is a continuous injective homomorphism of $\boldsymbol{R}^{2}$ into $\boldsymbol{K}^{3}$. Next we will show that $\left(X, \boldsymbol{R}^{3}\right)$ is minimal distal and $s\left(X, \boldsymbol{R}^{3}\right)>0$.

The flow $(X, \boldsymbol{R})$ is the usual imbedding of $\left(\boldsymbol{K}^{4}, S\right)$ and hence is minimal distal. It is easy to check that its maximal equicontinuous factor is $\left(\boldsymbol{K}^{2}, \boldsymbol{R}\right)$ when $((z, w), t) \rightarrow\left(e^{2 \pi i a t} z, e^{2 \pi i t} w\right)$ and $e^{2 \pi i a}=\alpha$. Let $Q($,$) denote the regional proximal relation. Then clearly$ $Q(X, \boldsymbol{R}) \subset Q\left(X, \boldsymbol{R}^{3}\right) \subset Q\left(X, \boldsymbol{K}^{3} \times \boldsymbol{R}\right)$. Moreover, $Q\left(X, \boldsymbol{K}^{3} \times \boldsymbol{R}\right)=$ $Q(X, \boldsymbol{R})$ because $\boldsymbol{R}$ is a syndetic subgroup of $\boldsymbol{K}^{3} \times \boldsymbol{R}$. The same argument works with the proximal relation. Therefore, $\left(X, \boldsymbol{R}^{3}\right)$ is minimal distal and its maximal equicontinuous factor is not locally free which implies by Proposition $1.5 s\left(X, \boldsymbol{R}^{3}\right)>0$. In fact, it is obvious that $s\left(X, \boldsymbol{R}^{3}\right)=2$.

3. Local sections and shear. A closed subset $S$ of $X$ is called a local section for $\left(X, \boldsymbol{R}^{p}\right)$ at $x_{0} \in X$ if there exists an $\epsilon>0$ such 
that the restriction of the action to $S \times \bar{B}(\epsilon)$ is a homeomorphism onto a neighborhood of $x_{0}$ where $B(\epsilon)=\left\{v \in \boldsymbol{R}^{P}:|v|<\epsilon\right\}$. We will refer to $\epsilon$ as the radius of the local section even though it is not unique. Zippin first stated without proof in [8, page 204] that Whitney's proof of the existence of local sections for flows could be extended to locally free $\boldsymbol{R}^{p}$ actions. (Recently Thomas wrote down a detailed proof of this in his thesis [7] which is the only proof we have seen.)

Let $S$ be a local section at $x_{0}$ of radius $\epsilon$ and let $U$ be an open subset of $X$ such that $x_{0} \in U \subset S \bar{B}(\epsilon)$.] Then there exists an open set $S_{0}$ of $S$ containing $x_{0}$ and $\epsilon^{\prime} \in(0, \epsilon)$ such that $S_{0} B\left(\epsilon^{\prime}\right) \subset U$. Then it is easy to check that $\bar{S}_{0}$ is a local section of radius $\epsilon^{\prime}$ such that the restriction of $(x, v) \rightarrow x v$ to $S_{0} \times B\left(\epsilon^{\prime}\right)$ is a homeomorphism onto an open neighborhood of $x_{0}$ whose closure is $\bar{S}_{0} \bar{B}(\epsilon)$. We will always assume $S_{0}$ is such an open dense subset of $S$.

Theorem 3.1. Let $\left(X, \boldsymbol{R}^{p}\right)$ be locally free, let $H=V \oplus D \in \mathscr{S}$ where $V$ is a subspace and $D$ is a discrete subgroup, and let $S$ be a local section of radius $r$ at $x_{0}$. Then there exist $\delta>0$ and open neighborhoods $U$ and $W$ of $x_{0}$ in $S$ and $\overline{x_{0} H}$ such that $U \times(V \cap B(\delta))$ is homeomorphic to $W$.

Proof. Let $\left\{e_{1}, \cdots, e_{p}\right\}$ be a basis for $\boldsymbol{R}^{p}$ such that

$$
H=\left\{\sum_{i=1}^{m} s_{i} e_{i}+\sum_{j=m+1}^{p} n_{j} e_{j}: s_{i} \in R \quad \text { and } \quad n, \in Z\right\} .
$$

In particular, $V$ is the span of $\left\{e_{1}, \cdots, e_{m}\right\}$ and $D$ is generated by $\left\{e_{m+1}, \cdots, e_{p}\right\}$. We will find it convenient to use the norm given by

$$
|v|=\left|\sum_{i=1}^{p} \lambda_{i} e_{\imath}\right|=\max _{1 \leqq i \leqq p}\left|\lambda_{l}\right|
$$

Set $K=\left\{\sum_{j=m+1}^{p} s_{j} e_{j}:\left|s_{l}\right| \leqq 1 / 2\right\}$. Clearly $K$ is compact, $K \cap V=$ $0,-K=K$ and $K+H=\boldsymbol{R}^{p}$.

LEMMA 3.2. For any $y \in X, y K \cap \overline{x_{0} H}$ is a finite nonempty set.

Proof. Since $\left(X, \boldsymbol{R}^{p}\right)$ is minimal, there exists $v_{n}=h_{n}+k_{n}, h_{n} \in H$, $k_{n} \in K$ such that $x_{0} v_{n} \rightarrow y$. We can also assume that $k_{n} \rightarrow k$ and $x_{0} h_{n} \rightarrow x$. Then $y=x k$ or $y(-k)=x \in \overline{x_{0} H}$ showing that $y K \cap \overline{x_{0} H} \neq \varnothing$. Suppose $y K \cap \overline{x_{0} H}$ is infinite and thus contains a convergent sequence $y k_{n} \rightarrow y k$ with $k_{n} \rightarrow k$ in $K$ and $k_{n} \neq k$. It follows that $k_{n}-k \rightarrow 0, \quad y k\left(k_{n}-k\right)=y k_{n} \in \overline{x_{0} H}=\overline{y k H}$, and $k_{n}-k \in H$ which is impossible. This completes the proof of the lemma. 
LEMMA 3.3. There exists a neighborhood $N$ of $x_{0}$ and a continuous function $F: N \rightarrow K$ such that $y F(y)=y K \cap \overline{x_{0} H}$ for all $y \in N$.

Proof. Suppose $x_{n} \rightarrow x_{0}$ and $x_{n} k_{n} \in \overline{x_{0} H}$ with $k_{n} \in K$. If $k_{n}$ does not converge to 0 , we can assume by taking a subsequence that $k_{n} \rightarrow k \neq 0$ which implies that $x_{0} k \in \widehat{x_{0} H}$ and $k \in H$. This contradicts the fact that $K \cap H=0$ and so $k_{n} \rightarrow 0$.

It follows that there exists a neighborhood $N$ of $x_{0}$ such that if $y \in N$ and $y k \in \overline{x_{0} H}$ for $k \in K$, then $|k|<1 / 4$. Consequently for $y \in N$ there is exactly one $k \in K$ such that $y k \in \overline{x_{0} H}$ and this defines $F$. Using sequences one routinely check that $F$ is continuous to complete the proof of the lemma.

Returning to the proof of the theorem we choose $U$ an open subset of $x_{0}$ in $S$ and $\epsilon>0$ such that $\epsilon$ is less than both radius of the cross section and $1 / 4, U B(\epsilon) \subset N$, and $|F(x)|<\epsilon / 2$ on $U$. Set $W^{\prime}=\{(x, s): x \in U$ and $|F(x)-s|<\epsilon / 2\}$ and note that it is open in $S \times \bar{B}(r)$. Hence $W^{\prime \prime}=\left\{x s:(x, s) \in W^{\prime}\right\}$ is open in $X$ and $W=W^{\prime \prime} \cap \overline{x_{0} H}$ is an open neighborhood of $x_{0}$ in $\overline{x_{0} H}$. It remains to show that $W$ is homeomorphic to $U \times(V \cap B .(\epsilon / 2))$. To do this first observe that $U \times(V \cap B(\epsilon / 2))$ is homeomorphic to $A=\{x(F(x)+v): x \in U, v \in V$, and $|v|<\epsilon / 2\}$ by mapping $(x, v) \rightarrow(x, v+F(x)) \rightarrow x(v+F(x))$, which reduces the problem to checking that $A=W$. Clearly $A \subset W$. If $x \in W$, then $x=y s$ where $y \in U$ and $|F(y)-s|<\epsilon / 2$. Writing $s=v+v^{\prime}$ where $v \in V$ and $v^{\prime}$ is in the span of $D$ we see that $|v|<\epsilon / 2$ and $\left|v^{\prime}\right|<1 / 4$ implying $v^{\prime} \in K$. Now $y v^{\prime} \in \overline{x_{0} H}(-v)=\overline{x_{0} H}, v^{\prime} \in K$, and $U \subset N$ imply that $v^{\prime}=F(y), x=y s=y(F(y)+v) \in A$, and $W \subset A$.

Corollary 3.4. Let $\left(X, \boldsymbol{R}^{p}\right)$ be locally free, let $x_{0} \in X$, and let $H=V \oplus D \in \mathscr{S}$. There exists a local section $S \subset \overline{x_{0} H}, \delta>0$, and an open set $S_{0}$ of $S$ such that $(y, v) \rightarrow y v$ is a homeomorphism of $S_{0} \times$ $(V \cap B(\delta))$ onto an open neighborhood of $x_{0}$ in $\overline{x_{0} H}$.

Proof. In the proof of the previous theorem it is easy to check that $S^{\prime}=\{x F(x): x \in \bar{U}\}$ is a section at $x_{0}$ in $\overline{x_{0} H}$. Now apply the theorem to $S^{\prime}$ noting that $F(x)=0$ in a neighborhood of $x_{0}$ in $S^{\prime}$.

Corollary 3.5. If $X$ is a differentiable manifold, $\left(X, \boldsymbol{R}^{p}\right)$ is a differentiable transformation group, and $H \in \mathscr{S}$, then $\overline{x_{0} H}$ is at least a topological manifold whose dimension is $\operatorname{dim} X-p+\operatorname{dim} H$.

4. Codimension one flows with zero shear. In this section $X$ will always be a compact $n$-dimensional manifold and a locally free transformation group $\left(X, \boldsymbol{R}^{n-1}\right)$ will be called a codimension one flow. As in the previous sections we will assume that $\left(X, R^{n-1}\right)$ is minimal. The following lemma will play a key role in this section: 
LEMMA 4.1. Let $\left(X, \boldsymbol{R}^{n-1}\right)$ be a codimension one flow and let $x_{0} \in X$. Then there exists a local section $S$ at $x_{0}$ and a homeomorphism $\varphi$ of $[-1,1]$ onto $S$ such that $\varphi(0)=x_{0}$.

Proof. Let $S$ be a local cross section at $x_{0}$ of radius $\epsilon$. Clearly the map $\theta: x s \rightarrow x$ is a continuous open map of $X \bar{B}(\epsilon)$ onto $S$. Let $D \subset S \bar{B}(\epsilon)$ be a compact neighborhood of $x_{0}$ which is homeomorphic to an $n$-dimensional disk. Let $S_{0}=\theta(\operatorname{Int} D)$. Then $\bar{S}_{0}=\theta(D)$ is an arcwise connected neighborhood of $x_{0}$ in $S$. Replacing $S$ by $\bar{S}_{0}$ allows us to assume without loss of generality that $S$ is arcwise connected. Next let $\gamma:[0,1] \rightarrow S$ be a homeomorphism. Consequently $(t, v) \rightarrow \gamma(t) v$ is a homeomorphism of $(0,1) \times B(\epsilon)$ into $X$ to which we can apply invariance of domain. It follows that $\gamma([0,1])$ is a local section of radius $\epsilon$ and $\gamma((0,1))$ is an open subset of $S$. For some $s_{0}, x_{0} s_{0} \in \gamma((0,1))$ from which it follows that $t \rightarrow \gamma(t)\left(-s_{0}\right)$ is a local section at $x_{0}$ of the desired kind.

This lemma holds without the assumption of minimality. With a little more work one can show that $x_{0}$ cannot be an endpoint of every arc in $S$ [1].

THEOREM 4.2. If $\left(X, \boldsymbol{R}^{n-1}\right)$ is a codimension one flow with $s\left(X, \boldsymbol{R}^{n-1}\right)=0$, then $\left(X, \boldsymbol{R}^{n-1}\right)$ is equicontinuous and $X$ is homeomorphic to $\boldsymbol{K}^{n}$.

Proof. There exists $H \in \mathscr{S}$ such that $\operatorname{dim} H=0$. From Theorem 3.1 and Lemma 4.1 it follows that $\overline{x_{0} H}$ is a compact one-dimensional manifold with a finite number of components $C_{0}, \cdots, C_{r}$. Let $H_{0}=$

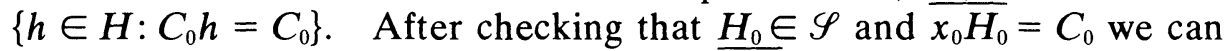
assume without loss of generality that $\overline{x_{0} H}$ is a compact connected one-dimensional manifold, i.e. a homeomorph of $K$. Since $H$ is isomorphic to the direct sum of $n-1$ copies of $Z,\left(\overline{x_{0} H}, H\right)$ is isomorphic to some $(\boldsymbol{K}, \boldsymbol{Z} \oplus \cdots \oplus \boldsymbol{Z})$. The next lemma reduces the theorem to showing that $\left(\overline{x_{0} H}, H\right)$ is equicontinuous and the subsequent one completes the proof by showing that every minimal transformation group of the form $(\boldsymbol{K}, \boldsymbol{Z} \oplus \cdots \oplus \boldsymbol{Z})$ is equicontinuous.

LEMMA 4.3. Let $\left(Y, \boldsymbol{R}^{p}\right)$ be a transformation group (not necessarily minimal) on a compact Hausdorff space, let $H$ be a closed syndetic subgroup of $\boldsymbol{R}^{p}$, and let $K$ be a compact subset of $\boldsymbol{R}^{p}$ such that $H+K=$ $\boldsymbol{R}^{p}$. If $M$ is a closed $H$ invariant subset of $Y$ such that (a) $(M, H)$ is equicontinuous, (b) $y \in M$ and $y s \in M$ implies $s \in H$, and (c) $y K \cap$ $M \neq \varnothing$ for all $y \in Y$, then $\left(Y, \boldsymbol{R}^{p}\right)$ is equicontinuous.

The proof is straightforward but a little tedius, so we omit it. The details can be found in [1]. 
LEMMA 4.4. If $(\boldsymbol{K}, \boldsymbol{Z} \oplus \cdots \oplus \boldsymbol{Z})$ is minimal, then there exists a homeomorphism $\psi$ of $\boldsymbol{K}$ onto $\boldsymbol{K}$ and a homomorphism $\theta$ of $\boldsymbol{Z} \oplus \cdots \oplus \boldsymbol{Z}$ into $K$ such that $\psi(z d)=\theta(d) \cdot \psi(z)$ ( $\cdot$ denotes complex multiplication) for all $d \in \boldsymbol{Z} \oplus \cdots \oplus \boldsymbol{Z}$ and $z \in \boldsymbol{K}$. In particular, $(\boldsymbol{K}, \boldsymbol{Z} \oplus \cdots \oplus \boldsymbol{Z})$ is equicontinuous.

Proof. Let $d_{1}, \cdots, d_{r}$ be the standard generator for $\boldsymbol{Z} \oplus \cdots \oplus \boldsymbol{Z}$. We can assume that $z\left(n d_{1}\right) \neq z$ for all $n \neq 0$ and $z \in$ $K$. Consequently there exists a unique minimal set $M$ under $z \rightarrow z d_{1}$. Since $\left(z d_{1}\right) d_{i}=\left(z d_{i}\right) d_{i}, z \rightarrow z d_{i}$ can be thought of as an automorphism of $z \rightarrow z d_{1}$ implying $M d_{i}=M$ for all $i$. Therefore, $\boldsymbol{M}=\boldsymbol{K}$ and there exists a homeomorphism $\psi$ of $\boldsymbol{K}$ onto $\boldsymbol{K}$ and a generator $\alpha_{1}$ of $\boldsymbol{K}$ such that $\psi\left(z d_{1}\right)=\alpha_{1} \cdot \psi(z)$. Because the only automorphisms of $\left(K, \alpha_{1}\right)$ are rotations, it follows that there exist $\alpha_{i}$ such that $\psi\left(z d_{i}\right)=\alpha_{i} \cdot \psi(z)$. Extending $d_{i} \rightarrow \alpha_{i}$ to a homomorphism $\theta$ of $\boldsymbol{Z} \oplus \cdots \oplus \boldsymbol{Z}$ into $\boldsymbol{K}$ we have $\psi(z d)=\theta(d) \cdot \psi(z)$.

In light of Theorem 4.2 , it is natural to ask if there are any dynamical properties which imply zero shear in the codimension one case and hence do not occur in an essential way for codimension one flows. For example, it is trivial that a locally free almost automorphic action of $\boldsymbol{R}^{p}$ has no shear. Thus a codimension one flow is almost automorphic if and only if it is equicontinuous. In our last theorem we prove a similar result for the distal case.

TheOREM 4.5. If $\left(X, \boldsymbol{R}^{n-1}\right)$ is a distal codimension of flow with trivial isotropy, then it is equicontinuous.

Proof. By Theorem 4.2 it suffices to show that $s\left(X, R^{n-1}\right)=$ 0 . Assume this is not true and let $H \in \mathscr{S}$ such that $0<\operatorname{dim} H=s$. By Theorem 3.1 and Lemma 4.1, $\overline{x_{0} H}$ is a compact manifold of dimension $s+1$. As before we can assume that $x_{0} H$ is connected. Let $(E, H)$ be the maximal equicontinuous factor of $\left(x_{0} H, H\right)$. Then $E$ is connected and non-trivial because $\overline{x_{0} H}$ is connected and minimal distal; in addition, the canonical projection $p$ of $\overline{x_{0} H}$ onto $E$ is open [3]. Taking advantage of the group structure of $(E, H)$ we obtain an open map $p^{\prime}$ of $\overline{x_{0}} H$ onto $K$ and a character $\Gamma$ on $H$ such that $p^{\prime}(x h)=\Gamma(h) \cdot p^{\prime}(x)$. Define $(K, H)$ by $(z, h) \rightarrow \Gamma(h) \cdot z$. Thus $p^{\prime}$ is an open homomorphism of $\left(\overline{x_{0} H}, H\right)$ onto $(K, H)$, and the proof will be completed by showing that such a homomorphism cannot exist with $\operatorname{dim} H=s>0$.

We will first show that $V \subset \operatorname{ker} \Gamma$. Assume $V \not \subset \operatorname{ker} \Gamma$. Then $\Gamma$ is onto; $\operatorname{ker} \Gamma$ is syndetic in $H$ and hence in $\boldsymbol{R}^{p}$; and $\operatorname{dim} \operatorname{ker} \Gamma<$ $\operatorname{dim} H$. Suppose $x_{0} h \in \overline{x_{0} \operatorname{ker} \Gamma}$. Clearly $h \in H$ because $\operatorname{ker} \Gamma \subset H$ and by applying $p^{\prime}$ we get $\Gamma(h) \cdot p^{\prime}\left(x_{0}\right)=p^{\prime}\left(x_{0}\right)$ or $\Gamma(h)=1$. We have shown that $\operatorname{ker} \Gamma \in \mathscr{S}\left(X, \boldsymbol{R}^{n-1}\right)$ which contradicts our choice of $H$. Therefore, $V \subset \operatorname{ker} \Gamma$. 
Since $\left(\overline{x_{0} H}, V\right)$ is distal, $(\overline{x V}, V)$ is a distal minimal set for all $x \in \overline{x_{0} H}$ which is nowhere dense in $\overline{x_{0} H}$ because $p^{\prime}(\overline{x V})=p^{\prime}(x)$ and $p^{\prime}$ is open. Let $S$ be a local section at $x_{0}$ given by Lemma 4.1 and Corollary 3.2. If $U$ is an open subset of $S$ contained in $S_{0}$, e.g. an open interval, then $p^{\prime}(U(V \cap B(\delta)))=p^{\prime}(U)$ is open in $\boldsymbol{K}$. Consequently $\overline{x V} \cap S$ is a closed nowhere dense set of $S$ which is self dense because $I\left(X, R^{n-1}\right)=$ 0 . Let $U$ be an arbitrarily small complementary open interval for $\overline{x V} \cap S$. Then $p^{\prime}(U)$ is open and $\overline{p^{\prime}(U)}=p^{\prime}(U) \cup p^{\prime}(x)$ which implies $p^{\prime}(U)=\boldsymbol{K} \backslash\left\{p^{\prime}(x)\right\}$. Finally since $U$ was arbitrarily small this contradicts the uniform continuity of $p^{\prime} \mid S$ and completes the proof.

In the context of minimal $\boldsymbol{R}^{p}$ actions on manifolds this result is sharp. From the example in $\$ 2$ we know there exist minimal distal codimension two flows with trivial isotropy which have non-zero shear and are not equicontinuous. Furthermore, if we carry out the same construction starting with $\boldsymbol{K}^{3}$ instead of $\boldsymbol{K}^{4}$, we will get a distal minimal codimension one flow with nontrivial isotropy which has nonzero shear and is not equicontinuous.

Corollary 4.6. Let $\left(X, \boldsymbol{R}^{n-1}\right)$ be a codimension one flow with trivial isotropy. Then the following are equivalent:

(a) $\left(X, \boldsymbol{R}^{n-1}\right)$ is distal

(b) $s\left(X, R^{n-1}\right)=0$

(c) $\left(X, \boldsymbol{R}^{n-1}\right)$ is equicontinuous.

(d) $\left(X, R^{n-1}\right)$ is almost automorphic.

Moreover, if any one of the conditions hold, then $X$ is homomorphic to $\boldsymbol{K}^{n}$.

\section{REFERENCES}

1. D. De Riggi, The structure of codimension one flows, Dissertation, University of Maryland, 1976.

2. R. Ellis and H. Keynes, A characterization of the equicontinuous structure relation, Trans. Amer. Math. Soc., 161 (1971), 171-183.

3. H. Furstenberg, The structure of distal flows, Amer. J. Math., 85 (1963), 477-515.

4. W. Gottschalk and H. Hedlund, Topological Dynamics, Amer. Math. Soc., Colloquium Publications, Vol. 36, 1955.

5. H. Keynes and D. Newton, Ergodic measures for non-abelian compact group extensions, Composition Math., 32 (1976), 53-70.

6. N. Markley, Local almost periodic minimal sets on the torus, Technical Report, (68-84), University of Maryland, 1968.

7. R. Thomas, Commuting continuous flows, Dissertation, University of Southampton, 1968.

8. L. Zippin, Transformation groups, in Lectures in Topology, University of Michigan Press, Ann Arbor, 1941, 191-221.

Received September 14, 1976 and in revised form January 31, 1977. The second author was supported by the National Science Foundation (MPS75-07078). 



\section{PACIFIC JOURNAL OF MATHEMATICS \\ EDITORS}

RICHARD ARENS (Managing Editor)

University of California

Los Angeles, CA 90024

\section{R. A. Beaumont \\ University of Washington \\ Seattle, WA 98105}

C. C. MOORE

University of California

Berkeley, CA 94720

\section{J. DUGUNDJI}

Department of Mathematics

University of Southern California

Los Angeles, CA 90007

R. FINN AND J. MILGRAM

Stanford University

Stanford, CA 94305

\section{ASSOCIATE EDITORS}

\section{E. F. BECKENBACH}

B. H. NeUmanN

F. WOLF

K. YoSHIDA

\section{SUPPORTING INSTITUTIONS}

\author{
UNIVERSITY OF BRITISH COLUMBIA \\ CALIFORNIA INSTITUTE OF TECHNOLOGY \\ UNIVERSITY OF CALIFORNIA \\ MONTANA STATE UNIVERSITY \\ UNIVERSITY OF NEVADA \\ NEW MEXICO STATE UNIVERSITY \\ OREGON STATE UNIVERSITY \\ UNIVERSITY OF OREGON \\ OSAKA UNIVERSITY
}

\author{
UNIVERSITY OF SOUTHERN CALIFORNIA \\ STANFORD UNIVERSITY \\ UNIVERSITY OF HAWAII \\ UNIVERSITY OF TOKYO \\ UNIVERSITY OF UTAH \\ WASHINGTON STATE UNIVERSITY \\ UNIVERSITY OF WASHINGTON \\ AMERICAN MATHEMATICAL SOCIETY
}

The Supporting Institutions listed above contribute to the cost of publication of this Journal, but they are not owners or publishers and have no responsibility for its contents or policies.

Mathematical papers intended for publication in the Pacific Journal of Mathematics should be in typed form or offset-reproduced (not dittoed), double spaced with large margins. Underline Greek letters in red, German in green, and script in blue. The first paragraph or two must be capable of being used separately as a synopsis of the entire paper. Items of the bibliography should not be cited there unless absolutely necessary, in which case they must be identified by author and Journal, rather than by item number. Manuscripts, in duplicate, may be sent to any one of the four editors. Please classify according to the scheme of Math. Reviews, Index to Vol. 39. All other communications should be addressed to the managing editor, or Elaine Barth, University of California, Los Angeles, California, 90024.

100 reprints are provided free for each article, only if page charges have been substantially paid. Additional copies may be obtained at cost in multiples of 50 .

The Pacific Journal of Mathematics is issued monthly as of January 1966. Regular subscription rate: $\$ 72.00$ a year (6 Vols., 12 issues). Special rate: $\$ 36.00$ a year to individual members of supporting institutions.

Subscriptions, orders for back numbers, and changes of address should be sent to Pacific Journal of Mathematics, 103 Highland Boulevard, Berkeley, California, 94708.

PUBLISHED BY PACIFIC JOURNAL OF MATHEMATICS, A NON-PROFIT CORPORATION

Printed at Jerusalem Academic Press, POB 2390, Jerusalem, Israel.

Copyright (C) 1977 Pacific Journal of Mathematics All Rights Reserved 


\section{Pacific Journal of Mathematics \\ Vol. 70, No. $2 \quad$ October, 1977}

B. Arazi, A generalization of the Chinese remainder theorem ........... 289

Thomas E. Armstrong, Polyhedrality of infinite dimensional cubes .... . . . 297

Yoav Benyamini, Mary Ellen Rudin and Michael L. Wage, Continuous

images of weakly compact subsets of Banach spaces ............ 309

John Thomas Burns, Curvature functions on Lorentz 2-manifolds ......... 325

Dennis F. De Riggi and Nelson Groh Markley, Shear distality and equicontinuity .................................. 337

Claes Fernström, Rational approximation and the growth of analytic

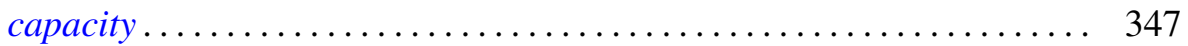

Pál Fischer, On some new generalizations of Shannon's inequality....... 351

Che-Kao Fong, Quasi-affine transforms of subnormal operators ......... 361

Stanley P. Gudder and W. Scruggs, Unbounded representations of

*-algebras........................................ 369

Chen F. King, A note on Drazin inverses .................... 383

Ronald Fred Levy, Countable spaces without points of first countability . . . 391

Eva Lowen-Colebunders, Completeness properties for convergence

spaces ......................................... 401

Calvin Cooper Moore, Square integrable primary representations ....... 413

Stanisław G. Mrówka and Jung-Hsien Tsai, On preservation of

E-compactness ................................ 429

Yoshiomi Nakagami, Essential spectrum $\Gamma(\beta)$ of a dual action on a von

Neumann algebra ................................ 437

L. Alayne Parson, Normal congruence subgroups of the Hecke groups

$G\left(2^{(1 / 2)}\right)$ and $G\left(3^{(1 / 2)}\right)$...

Louis Jackson Ratliff, Jr., On the prime divisors of zero in form rings . . . . 489

Caroline Series, Ergodic actions of product groups .................. 519

Robert O. Stanton, Infinite decomposition bases..................... 549

David A. Stegenga, Sums of invariant subspaces .................. 567 